\title{
Hydrogen-rich saline reduces cell death through inhibition of DNA oxidative stress and overactivation of poly (ADP-ribose) polymerase-1 in retinal ischemia-reperfusion injury
}

\author{
HONGWEI LIU ${ }^{*}$, NING HUA ${ }^{2 *}$, KELIANG XIE $^{1 *}$, TINGTING ZHAO $^{1}$ and YONGHAO YU ${ }^{1}$ \\ ${ }^{1}$ Department of Anesthesiology, Tianjin Institute of Anesthesiology, Tianjin Medical University General Hospital; \\ ${ }^{2}$ Department of Pediatric Ophthalmology and Strabismus, Tianjin Medical University Eye Hospital, Tianjin 300052, P.R. China
}

Received July 10, 2014; Accepted March 24, 2015

DOI: $10.3892 / \mathrm{mmr} .2015 .3731$

\begin{abstract}
Overactivation of poly (ADP-ribose) polymerase 1 (PARP-1), as a result of sustained DNA oxidation in ischemia-reperfusion injury, triggers programmed cell necrosis and apoptosis. The present study was conducted to demonstrate whether hydrogen-rich saline (HRS) has a neuroprotective effect on retinal ischemia reperfusion (RIR) injury through inhibition of PARP-1 activation. RIR was induced by transient elevation of intraocular pressure in rats. HRS $(5 \mathrm{ml} / \mathrm{kg})$ was administered peritoneally every day from the beginning of reperfusion in RIR rats until the rats were sacrificed. Retinal damage and cell death was determined using hematoxylin and eosin and terminal deoxynucleotidyl transferase dUTP nick end labeling staining. DNA oxidative stress was evaluated by immunofluorescence staining of 8-hydroxy-2-deoxyguanosine. In addition, the expression of PARP-1 and caspase- 3 was investigated by western blot analysis and/or immunohistochemical staining. The results demonstrated that HRS administration improved morphological alterations and reduced apoptosis following RIR injury. Furthermore, the present study found that HRS alleviated DNA oxidation and PARP-1 overactivation in RIR rats. HRS can protect RIR injury by inhibition of PARP-1, which may be involved in DNA oxidative stress and caspase-3-mediated apoptosis.
\end{abstract}

Correspondence to: Dr Yonghao Yu, Department of Anesthesiology, Tianjin Institute of Anesthesiology, Tianjin Medical University General Hospital, 154 Anshan Road, Tianjin 300052, P.R. China

E-mail: 58159529@qq.com

${ }^{*}$ Contributed equally

Key words: oxidative stress, retinal ischemia-reperfusion injury, poly (ADP-ribose) polymerase-1, hydrogen-rich saline

\section{Introduction}

Retinal ischemia reperfusion (RIR) injury exists in various eye diseases, including glaucoma, diabetic retinopathy and other ocular vascular disorders (1-3). Production of reactive oxygen species (ROS) in RIR induces oxidative stress, which leads to lipid oxidation, protein synthetic disorder and DNA oxidation $(4,5)$. It has been verified that nuclear and mitochondrial DNA oxidation leads to neuron death (6-8). Therefore, the prevention of DNA injury may be an effective strategy to promote retinal cellular survival.

ROS, particularly peroxynitrite, can induce nuclear DNA oxidative breaks, followed by activation of poly (ADP-ribose) polymerase-1 (PARP-1), a nuclear enzyme involved in the regulation of multiple pathophysiological cellular procedures, including DNA repair, gene transcription and cell death (9-11). PARP-1 catalyzes the formation of poly (ADP-ribose) polymers, which triggers the translocation of apoptosis-inducing factor (AIF) from the mitochondria into the nucleus, causing DNA condensation and caspase-independent cell death. In addition, ROS induced mitochondrial DNA stress and lipid oxidation and can also induce mitochondrial structural breakdown and the release of cytochrome $c$, which promotes caspase family activation and apoptotic cell death $(8,12)$.

Hydrogen, the most common gas in the atmosphere, was initially recognized as a therapeutic reductive substance in medicine in 1975 (13). Several studies (14-17) regarding its usage and underlying molecular mechanism have been conducted since Ohsawa et al identified that the inhalation of hydrogen gas markedly suppressed ischemia-reperfusion injury in the brain by alleviating oxidative stress caused by ROS in 2007 (18). Hydrogen can be dissolved in water up to $0.8 \mathrm{mM}$ under atmospheric pressure at room temperature and its solubilized form, hydrogen-rich saline (HRS), is beneficial since it is a portable, easily administered and a safe means of delivering hydrogen (14). In addition, administration of HRS has been demonstrated to enhance cell survival in different disorders in animal models, particularly due to its anti-oxidative and anti-inflammatory properties (15-17).

The present study aimed to investigate whether HRS had a protective effect in rodent RIR injury. In addition, the mechanism underlying the protective effects of HRS was investigated 
by measuring DNA oxidative stress, PARP-1 expression and apoptosis.

\section{Materials and methods}

Animals. A total of 483 month-old adult male Sprague-Dawley rats weighing 300-350 g (Tianjin Medical University Animal Center, Tianjin, China) were used in the present study. Animals were fed a standard rodent diet with a normal light-dark cycle. All animals were cared for with the approval of the Laboratory Animal Research Committee at Tianjin Medical University Eye Center. All experimental procedures involving animals were performed in accordance with the ARVO Statement for the Use of Animals in Ophthalmic and Vision Research.

HRS production. Hydrogen was dissolved and saturated in $0.9 \%$ normal saline (NS) for $6 \mathrm{~h}$ under a high pressure of $0.4 \mathrm{MPa}$ using hydrogen-producing apparatus (GCH-3000; Tianjin Tongpu Analytical Instrument Tech Co., Ltd., Tianjin, China) according to the method reported by Cai et al (17). The saturated HRS was stored at $4^{\circ} \mathrm{C}$ under normal pressure in an aluminum bag. HRS was freshly prepared and sterilized by $\gamma$ radiation $24 \mathrm{~h}$ prior to the experiment, ensuring an effective concentration of $0.6 \mathrm{mM}$. The content of hydrogen in NS was confirmed through gas chromatography as described previously by Ohsawa et al (14).

Rat RIR model and peritoneal administration of HRS. All surgery was performed under general anesthesia with intraperitoneal administration of chloral hydrate $(300 \mathrm{mg} / \mathrm{kg})$. The anterior chamber of the left eye was cannulated with a 30 -gauge needle connected to a reservoir containing NS. Intraocular pressure was increased to $130 \mathrm{mmHg}$ for $60 \mathrm{~min}$ and ocular ischemia was confirmed by interruption of the ocular circulation, as described by Sun et al (2). Thereafter, the cannula was immediately retracted and the adequacy of retinal reperfusion was confirmed visually by ophthalmoscopy. Rats that did not recover from retinal perfusion $3 \mathrm{~min}$ after the end of the ischemic period and those with lens injury, which prevents retinal ganglion cell (RGC) death and promotes axonal regeneration (19), were excluded from the investigation. Following surgery, levofloxacin ophthalmic solution $0.3 \%$ (Cravit ${ }^{\circledR}$; Santen Pharmaceutical Co., Ltd., Osaka, Japan) was dropped into the conjunctival sac of the left eye to prevent infection. The model of IR injury was fully induced $24 \mathrm{~h}$ after reperfusion.

All the rats were classified into the following four groups: IR injury with HRS group, IR injury with NS group, HRS-treated control group and NS-treated control group. The animals in the IR injury with HRS and NS groups were peritoneally injected with HRS or NS $(5 \mathrm{ml} / \mathrm{g})$ at the beginning of reperfusion. Consecutive HRS or NS treatment was administered daily until the rats were sacrificed with overdose anaesthesia. The rats in the HRS-treated and NS-treated control groups were peritoneally injected with HRS or NS $(5 \mathrm{ml} / \mathrm{kg})$ at the same time points without initial reperfusion. As molecular hydrogen can easily diffuse into tissues and penetrate the cellular membrane, HRS $(5 \mathrm{ml} / \mathrm{kg})$ was injected peritoneally only at the beginning of reperfusion.
Furthermore, consecutive HRS, compared with the equivalent quantity of NS, was administered daily until the rats were sacrificed.

Histological staining. Freshly enucleated eyeballs $(n=6)$ were fixed in $4 \%$ paraformaldehyde in $0.1 \mathrm{M}$ phosphate-buffered saline (PBS) for $30 \mathrm{~min}$. Subsequently, corneal paracentesis was performed using a $25 \mathrm{G}$ needle. Following that, the entire eye cups were further fixed overnight and then processed for paraffin embedding. Sections $(4 \mu \mathrm{m})$ were cut along the vertical meridian of the eye, $2 \mathrm{~mm}$ away from the optic nerve head and mounted on pre-coated glass slides. Following deparaffinization and rehydration, sections were stained with hematoxylin and eosin. Retinal damage was assessed by measuring the thickness of the retina and cell loss in the ganglion cell layer (GCL). The retinal thickness is defined as the total width between the inner limiting membrane to the interface of the outer plexiform layer and the outer nuclear layer (20). The number of cells in the GCL was calculated under a light microscope (magnification, x40) (BX51; Olympus Corporation, Tokyo, Japan). Three different sections were randomly selected and each section was measured at four different points between 1 and $2 \mathrm{~mm}$ from the optic disc. The average value was appointed as the thickness of retina and the number of cells in the GCL of the eye.

Terminal deoxynucleotidyl transferase dUTP nick end labeling (TUNEL). Cryosections $(\mathrm{n}=6)$ were fixed with $4 \%$ paraformaldehyde in 0.1 M PBS (pH 7.4) for $20 \mathrm{~min}$. Cell death was detected by a TUNEL assay using the In Situ Cell Death Detection kit, POD (Roche Diagnostics Deutschland $\mathrm{GmbH}$, Mannheim, Germany) and color-developed with 3,3'-diaminobenzidine (DAB) substrate (Roche Diagnostics Deutschland $\mathrm{GmbH}$ ) according to the manufacturer's instructions. The number of TUNEL-positive cells in the GCL was counted at $\mathrm{x} 400$ magnification for each section using a light microscope (BX51; Olympus Corporation).

Immunofluorescence staining of 8-hydroxy-2-deoxyguanosine $(8-O H d G)$. Eyes $(n=6)$ in optimal cutting temperature compound were cut into $6 \mu \mathrm{m}$ sections (RM2165; Leica, Wetzlar, Germany) following being flash frozen in liquid nitrogen. The cryosections were fixed in ice-cold acetone for 10 min, washed in PBS three times and blocked with $1 \%$ bovine serum albumin (BSA) in PBST for $30 \mathrm{~min}$ at room temperature. Subsequently, primary goat anti-8-hydroxyguanine $(8-\mathrm{OHdG}$, a classical DNA oxidative product) polyclonal antibody (1:100; cat. no. ab10802; Abcam, San Francisco, CA, USA) in 1\% BSA in PBST were added to the samples and incubated in a humidified chamber overnight at $4^{\circ} \mathrm{C}$. Following three washes in PBS, the sections were incubated with tetramethyl rhodamine isothiocyanate-conjugated rabbit anti-goat IgG secondary antibody (1:200; cat. no. BA1091; Beijing Zhongshan Golden Bridge Biotechnology Co., Ltd., Beijing, China) for $1 \mathrm{~h}$ in the dark at $37^{\circ} \mathrm{C}$, followed by 4',6-diamidino-2-phenylindole staining (0.1 $\mu \mathrm{g} / \mathrm{ml}$; Sigma-Aldrich, St. Louis, MO, USA) and another three washes in PBS. Coverslides were mounted and the immunoreactivity of $8-\mathrm{OHdG}$ was detected under a fluorescence microscope (DFC500; Leica Microsystems, Renens, Switzerland). 

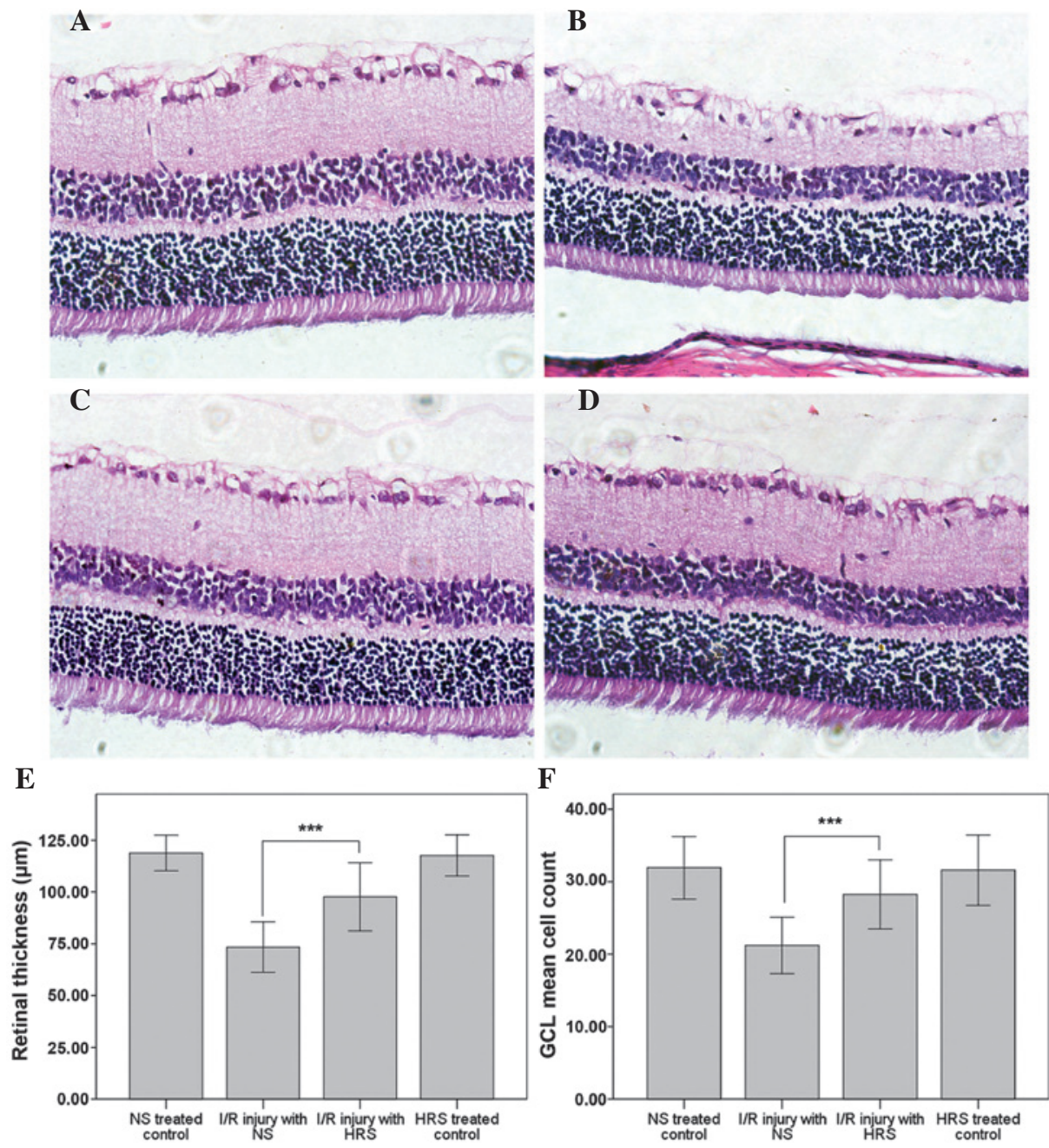

Figure 1. HRS improves histological impairment in a rat model of RIR (magnification, $x 400$ ). The retinas were sliced after paraffin embedding and stained with hematoxylin and eosin 1 week after RIR injury. (A) Images of representative sections from the NS-treated control group, (B) RIR injury with NS group, (C) the HRS-treated control group and (D) the RIR injury with HRS group are shown. (E) Retinal thicknesses following peritoneal administration of HRS. (F) Number of cells in the GCL following peritoneal administration of HRS. ${ }^{* * *} \mathrm{P}<0.0001$, compared with I/R-injured retina treated with NS. Histograms represent the mean \pm standard deviation. HRS, hydrogen-rich saline; RIR, retinal ischemia reperfusion; GCL, ganglion cells layer; NS, normal saline; I/R, ischemia/reperfusion.

Western blot analysis. Total retinal tissue lysates $(\mathrm{n}=6)$ were prepared by the addition of $500 \mu 1$ sodium dodecyl sulfate (SDS) buffer [250 mM Tris (pH 6.8), 10\% SDS, $500 \mathrm{mM}$ dithiothreitol, $50 \%$ glycerol, $0.5 \%$ bromophenol blue and a 1:100 protease inhibitor cocktail (cat. no. P8340; Sigma-Aldrich)]. Total tissue extracts $(20 \mu \mathrm{g})$ were separated on a $12 \%$ SDS polyacrylamide gel. Proteins were transferred onto an Immobilon-P membrane (Millipore, Billerica, MA, USA) and blocked with 5\% skim milk in TBST (TBS containing $0.05 \%$ Tween-20) at room temperature for $2 \mathrm{~h}$. Subsequently, the membranes were hybridized at $4^{\circ} \mathrm{C}$ overnight in TBST with the primary antibodies: Anti-rPARP-1 (1:5,000; cat. no. 1051-1; Epitomics, Burlingame, CA, USA), and anti-rCaspase-3 (1:1,000; cat. no. 9661; Cell Signaling Technology, Boston, MA, USA) overnight at $4^{\circ} \mathrm{C}$. Following incubation with a horseradish peroxidase-conjugated anti-rabbit immunoglobulin antibody, proteins were visualized with an enhanced chemiluminescence kit (Kirkegaard \& Perry Laboratories, Inc., Gaithersburg, MD, USA). For normalization, blots were probed with $\beta$-actin, a housekeeping antibody (anti- $\beta$-actin; 1:1,000; Beijing Zhongshan Golden
Bridge Biotechnology Co., Ltd.). The signal was detected by exposing X-ray films to the processed blots and analyzed by laser scanning densitometry (Personal Densitometer; GE Healthcare, Piscataway, NJ, USA).

Immunohistochemical staining of PARP-1. Paraffin-embedded sections ( $\mathrm{n}=6,4 \mu \mathrm{m}$ thick) were deparaffinized and rehydrated. Antigen retrieval was achieved by boiling in $10 \mathrm{mmol} / \mathrm{l}$ sodium citrate buffer for $10 \mathrm{~min}$ and then steadily cooling to room temperature. Subsequently, the sections were blocked using $3.0 \% \mathrm{H}_{2} \mathrm{O}_{2}$ in methanol for $15 \mathrm{~min}$ in order to inhibit endogenous peroxidase activity. Following washing in PBS, the sections were incubated overnight at $4^{\circ} \mathrm{C}$ with monoclonal rabbit anti-PARP-1 primary antibody (cat. no. 1051-1) at a dilution of 1:40 (Epitomics). The sections were incubated with peroxidase-conjugated goat anti-rabbit IgG (Beijing Zhongshan Goldenbridge Biotechnology Co., Ltd.) at the same dilution of 1:500 for $2 \mathrm{~h}$ at $37^{\circ} \mathrm{C}$. The sections were washed in PBS, developed in prepared $\mathrm{DAB}$ chromogen solution, lightly counterstained with hematoxylin, dehydrated and mounted. 

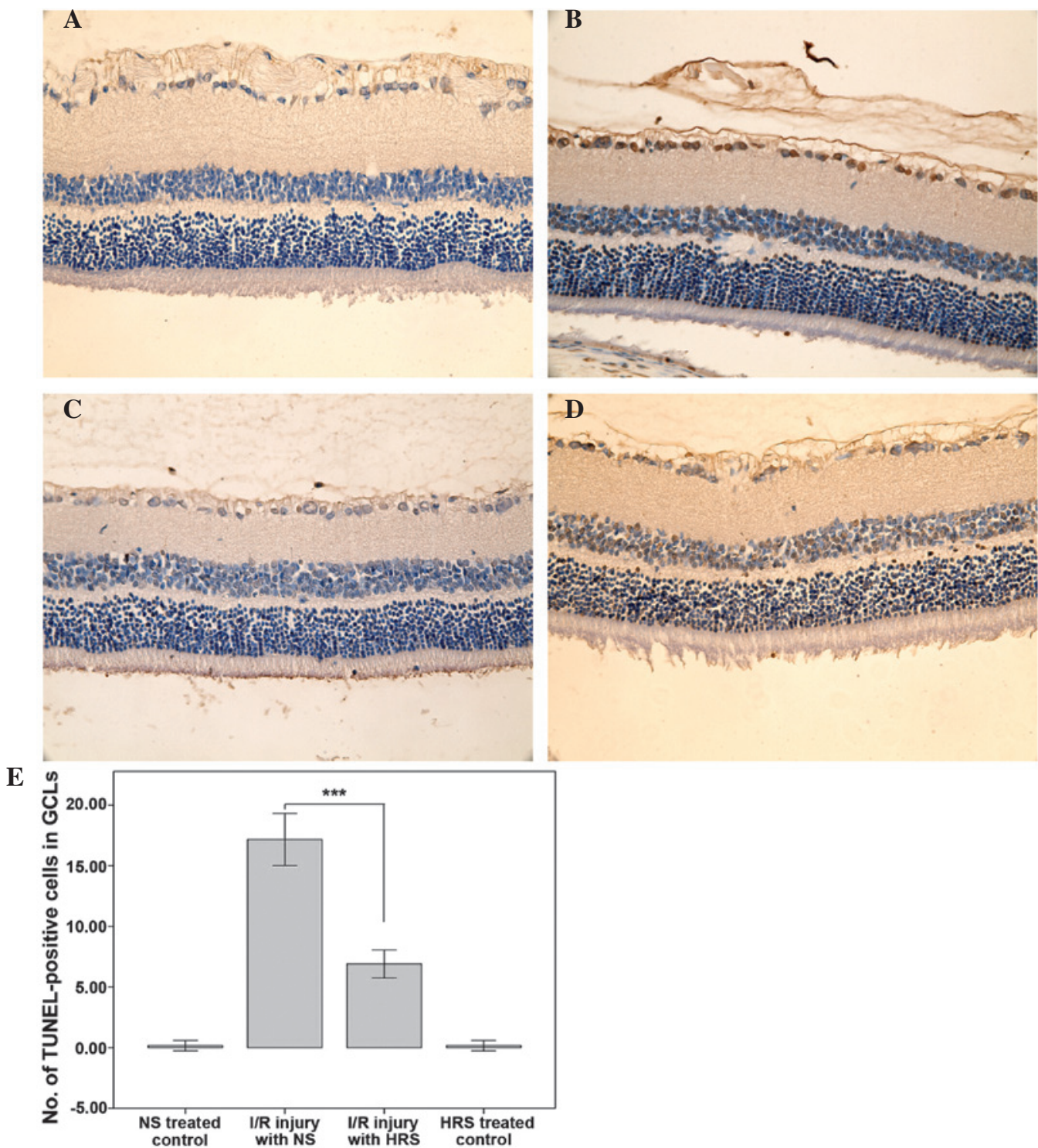

Figure 2. HRS alleviates cell death in the GCL in a rat model of RIR (magnification, $\mathrm{x} 400$ ). Retinal cryosections ( $\mathrm{n}=6$ ) were stained with TUNEL using the in situ cell death detection kit 1 week after RIR injury and cell death was detected as brown nuclei by 3,3'-diaminobenzidine staining. (A) Images of representative sections of the NS-treated control group, (B) RIR injury with NS group, (C) the HRS-treated control group and (D) the RIR injury with HRS group are shown. (E) The administration of HRS significantly alleviated cell death in the GCL. ${ }^{* * *} \mathrm{P}<0.0001$, compared with I/R-injured retina treated with NS. Histograms represent the mean \pm standard deviation. HRS, hydrogen-rich saline; RIR, retinal ischemia reperfusion; TUNEL, terminal deoxynucleotidyl transferase dUTP nick end labeling; GCL, ganglion cell layer; NS, normal saline; I/R, ischemia/reperfusion.

Statistical analysis. All data are presented as the mean \pm standard deviation. Data were analyzed using one-way analysis of variance conducted between groups with post hoc analysis through Bonferroni multiple comparisons test. Student's t-test was used to compare data between two groups. Statistical analyses were conducted using SPSS version 18.0 (SPSS Inc., Chicago, IL, USA). P $<0.05$ was considered to indicate a statistically significant difference.

\section{Results}

HRS administration reduces histological disorder following RIR injury. The histological alterations of RIR injury occurred after 1 week of reperfusion (21). The thinning of retinas and cell loss in the GCL demonstrated the successful induction of this model. Abdominal administration of HRS effectively reversed the morphological alterations, including thinning of retinas and cell loss in the GCL (Fig. 1). By contrast, the retinal thickness of the RIR injury with NS group was $73.39 \pm 6.07 \mu \mathrm{m}$, which was markedly thinner than the retinal thickness in the NS-treated control group $(118.85 \pm 4.31 \mu \mathrm{m} ; \mathrm{P}<0.001)$. The retinal thickness in the HRS-treated control group was $117.71 \pm 4.95 \mu \mathrm{m}$, which demonstrated that hydrogen did not alter the thickness of the retina in normal rats. However, following HRS administration the thinning of retina following RIR injury partially recovered to $97.72 \pm 8.25 \mu \mathrm{m}$, which was significantly different to the NS-treated control $(\mathrm{P}<0.001)$.

Furthermore, the number of cells in the GCL in the IR injury with NS group decreased to $21.2 \pm 1.9$, compared with $31.9 \pm 2.2$ in the NS-treated control $(\mathrm{P}<0.001)$. No significant difference was identified between the HRS-treated (31.6 \pm 2.4$)$ 

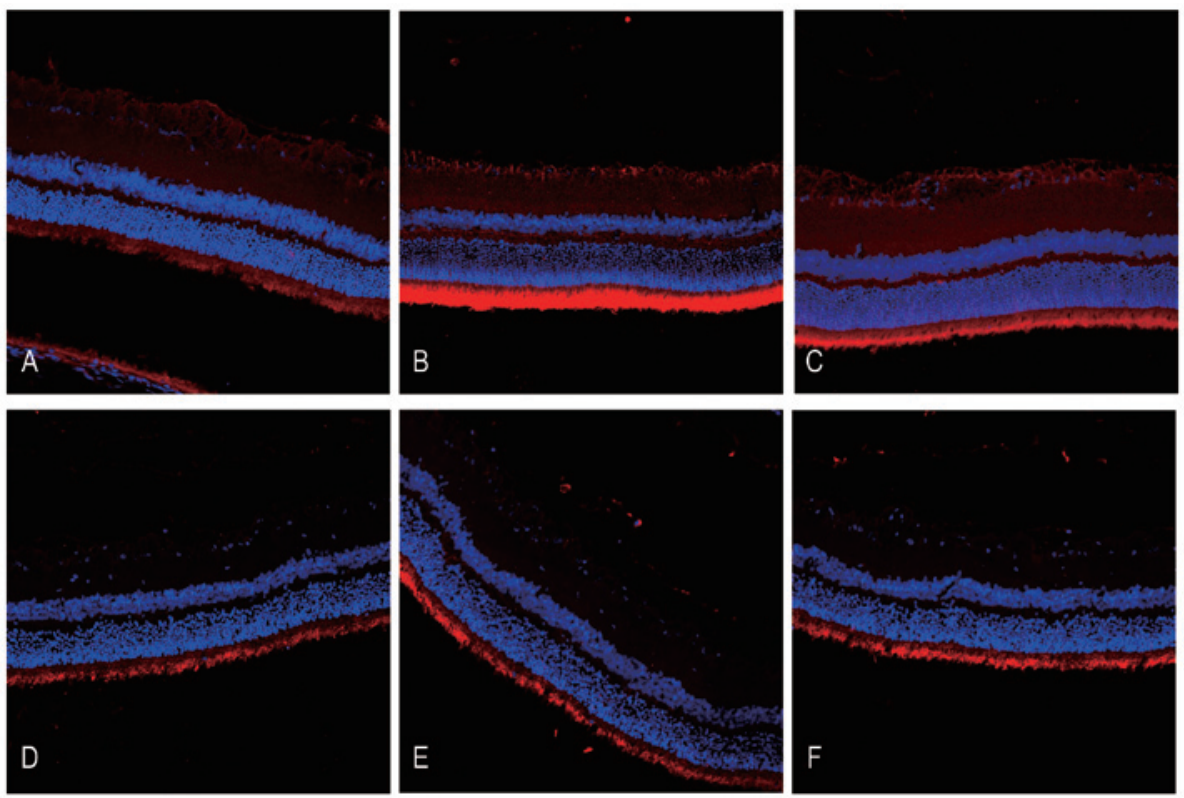

Figure 3. DNA oxidative injury detected by immunoreactivity of 8-OHdG is ameliorated following HRS intervention between $24 \mathrm{~h}$ and 7 days after reperfusion in a rat RIR model (magnification, $x 200)$. Retinal cryosections $(n=6)$ were stained with primary goat anti 8 -OHdG polyclonal antibody $(1: 100)$ overnight at $4^{\circ} \mathrm{C}$ and were incubated with tetramethyl rhodamine isothiocyanate-conjugated rabbit $\mathrm{IgG}$ secondary antibody (1:200 for $1 \mathrm{~h}$ in the dark at $37^{\circ} \mathrm{C}$, followed by 4',6-diamidino-2-phenylindole staining; $0.1 \mu \mathrm{g} / \mathrm{ml}$ ). The immunoreactivity of 8-OHdG was detected under a fluorescence microscope. (A) NS-treated control group, (B) RIR injury with NS group $24 \mathrm{~h}$ after reperfusion, (C) RIR injury with NS group 7 days after reperfusion, (D) HRS-treated control group, (E) RIR injury with HRS group $24 \mathrm{~h}$ after reperfusion and (F) the RIR injury with HRS group 7 days after reperfusion. HRS, hydrogen-rich saline; 8 OHdg, 8-hydroxy-2-deoxyguanosine; RIR, retinal ischemia reperfusion; NS, normal saline.

or NS-treated controls. By contrast, the number of cells in the IR injury with HRS group was 28.3 $\pm 2.4(\mathrm{P}<0.001)$.

$H R S$ administration alleviates death of RGCs in RIR injury. The NS-treated control and HRS-treated control had few TUNEL positive cells, which illustrated that HRS did not induce cell death. By contrast, retinas with RIR injury experienced severe cell death in the GCL 7 days after reperfusion, which was in accordance with previous studies $(22,23)$. Treatment with HRS significantly decreased the number of TUNEL-positive cells in the GCL of RIR rats on the seventh day after reperfusion $(\mathrm{P}<0.001)$, suggesting that HRS may have a protective effect on cell apoptosis (Fig. 2).

HRS administration alleviates DNA oxidative breaks in RIR injury. The DNA oxidative breaks in RIR injury were detected through immunofluorescent staining of $8-\mathrm{OHdG}$, a DNA oxidative product (Fig. 3). There was moderate 8-OHdG immunoreactivity in the photoreceptor cell layer compared with faint immunostaining in the GCL in the untreated control. Following administration of HRS, 8-OHdG immunoreactivity partially decreased. In addition, IR injury increased the strength of $8-\mathrm{OHdG}$ immunoreactivity in the GCL as well as in photoreceptor cell layers, suggesting severe DNA oxidative stress due to ROS. By contrast, the immunoreactivity of $8-\mathrm{OHdG}$ decreased significantly in the GCL immediately following HRS intervention in IR injury, which may confirm the reductive potential of hydrogen to DNA oxidative stress.

PARP-1 and cleaved PARP-1 are downregulated following hydrogen intervention, concordant with the expression of caspase-3. Excessive DNA breaks may induce overactivation

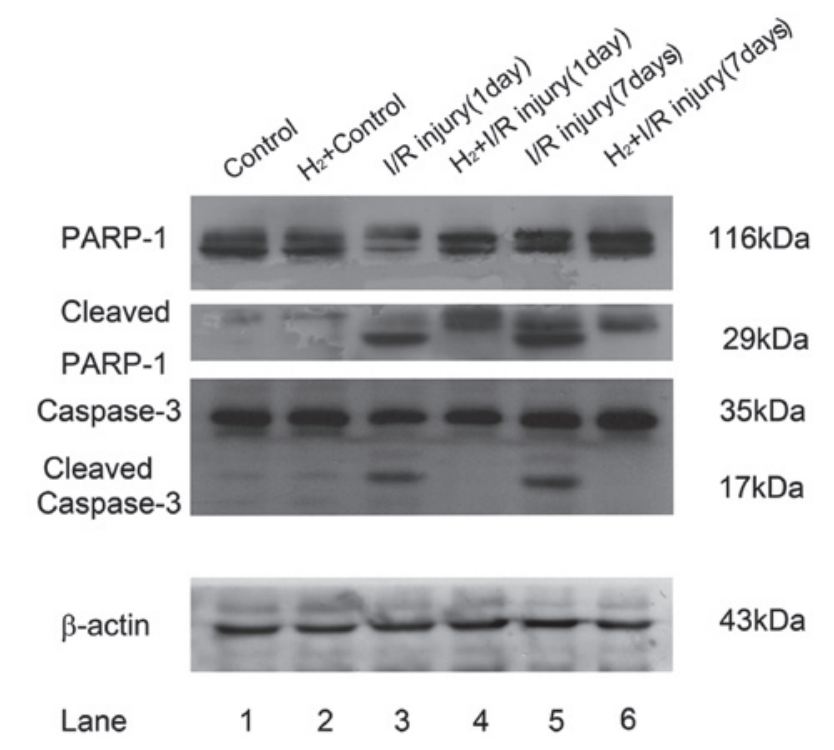

Figure 4. Alterations in PARP-1 and caspase-3 cleavage following administration of HRS in a retinal $\mathrm{I} / \mathrm{R}$ model in rats. Retinas $(n=6)$ were lysed with RIPA mixed with a protease inhibitor cocktail. Lysates $(20 \mu \mathrm{g})$ from each group were added into their corresponding layers of $12 \%$ SDS-PAGE. Following being transferred onto a polyvinylidene fluoride membrane, primary antibodies (anti-rPARP-1; 1:5,000; anti-rCaspase-3, $1: 1,000)$ were cultured at $4^{\circ} \mathrm{C}$ overnight followed by horseradish peroxidase-conjugated secondary antibody cultured for $2 \mathrm{~h}$ at $37^{\circ} \mathrm{C}$. Subsequently, electrochemiluminescnce-assisted exposure was performed to gain $116 \mathrm{kDa}$ full PARP-1 and its cleaved 29 kDa fragments, the intact caspase-3 (35 kDa) and its $17 \mathrm{kDa}$ cleaved fragment. For normalization, blots were probed with $\beta$-actin (anti- $\beta$-actin; 1:1,000). Compared with the control, RIR injury led to significant cleavage of full length PARP-1 between $24 \mathrm{~h}$ and 7 days after reperfusion (lane 1 and 2 versus lane 3 and 5). The cleavage of caspase- 3 had a similar change to PARP-1. HRS intervention of RIR injury significantly reduced the cleavage of these two proteins (lane 3 and 5 versus lane 4 and 6). PARP-1, poly (ADP-ribose) polymerase 1; HRS, hydrogen-rich saline; I/R, ischemia/reperfusion. 

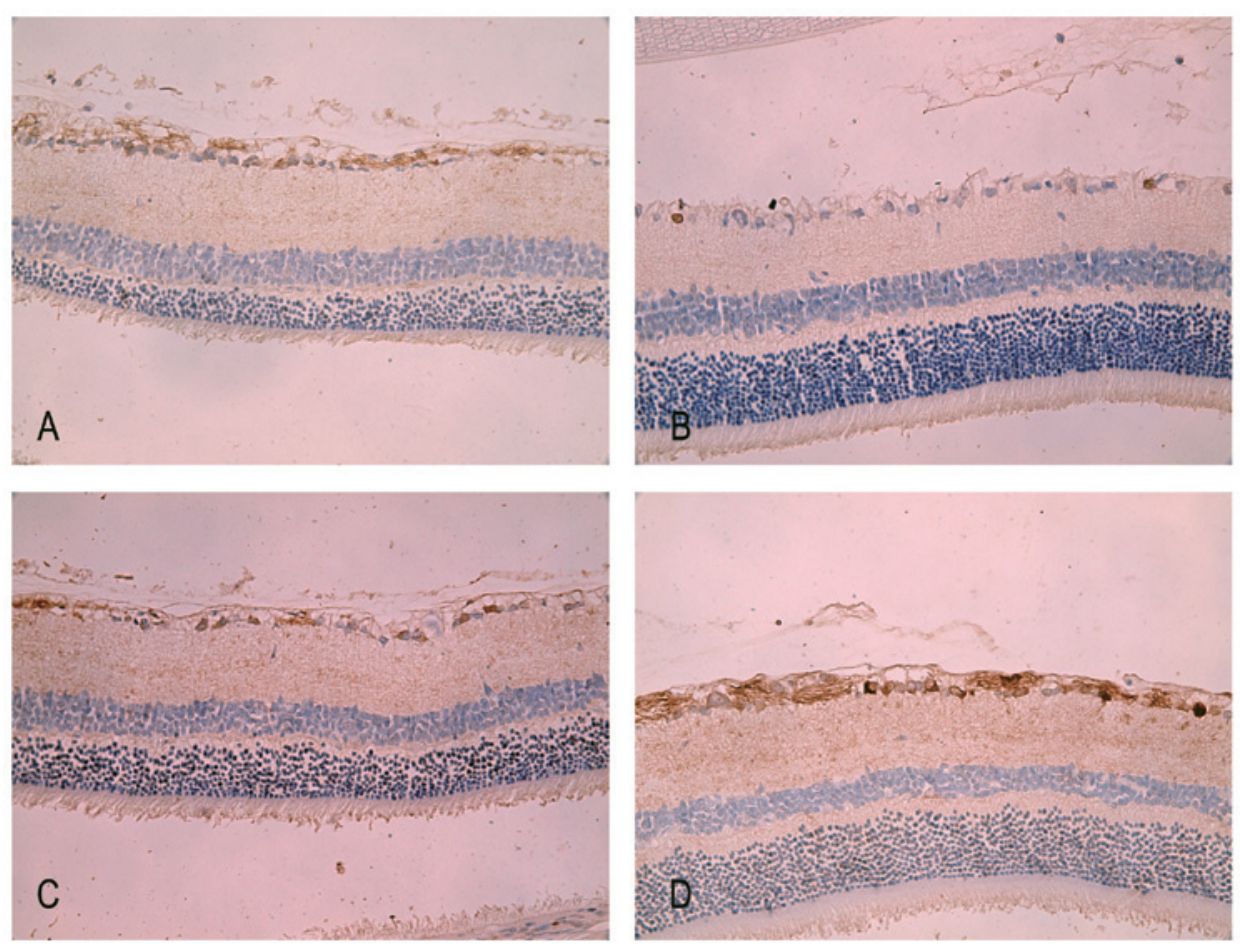

Figure 5. HRS affects the expression and sub-cellular distribution of PARP-1 in the ganglion cell layer in a rat RIR model (magnification, $\mathrm{x} 400)$. The retinas were sliced after paraffin embedding and stained with anti-rPARP-1 antibody (1:40) and anti-peroxidase-conjugated goat anti-rabbit IgG (1:500) 1 week after RIR injury. The sections were color-developed in 3,3'-diaminobenzidine and lightly counterstained with hematoxylin. (A) Images of representative sections from the NS-treated control group, (B) RIR injury with NS group, (C) HRS-treated control group and (D) RIR injury with HRS group are shown. The immunoreactivity of PARP-1 was concentrated in nuclei of the ganglion cell layer in the RIR group. However, it distributed extensively in the nerve fiber layer and ganglion cell layer following administration of HRS. HRS, hydrogen-rich saline; PARP-1, poly (ADP-ribose) polymerase 1; RIR, retinal ischemia reperfusion.

of PARP-1 and cell death. In addition, as the substrate of caspase-3, activated PARP-1 can be cleaved into 29 and $84 \mathrm{kDa}$ fragments. The primary anti-capase- 3 antibody could react with the whole molecule and the $19 \mathrm{kDa}$ fragment of caspase-3, while anti-PARP-1 antibody recognized full length PARP-1 and its $29 \mathrm{kDa}$ fragment.

Cleavage of PARP-1 was significantly elevated in RIR retina $24 \mathrm{~h}$ and 7 days after reperfusion (Fig. 4). Compared with the relatively low immunofluorescent staining of PARP-1 at $116 \mathrm{kDa}$ in the untreated control and hydrogen control, the RIR model demonstrated strong immunoreactivity of the $29 \mathrm{kDa}$-terminal catalytic domain. At the same time, the pro-form of PARP-1 decreased $24 \mathrm{~h}$ after RIR injury, but increased and surpassed the control 7 days after RIR injury, while the $29 \mathrm{kDa}$ cleaved fragment remained visible. Following peritoneal administration of HRS, cleaved PARP-1 was reduced $24 \mathrm{~h}$ and 7 days after reperfusion, demonstrated as decreased immunoreactivity of $29 \mathrm{kDa}$ PARP-1 fragments.

The expression of capase- 3 was detected in RIR injury by immunostaining. In the untreated control retina and hydrogen control retina, there was almost no caspase- 3 immunoreactivity. However, cleaved caspase- 3 was detected between $24 \mathrm{~h}$ and 7 days after reperfusion. However, hydrogen intervention inhibited caspase-3 cleavage, showing the loss of cleaved fragment immunoreactivity $24 \mathrm{~h}$ and 7 days after reperfusion.

Immunohistochemistry staining of PARP-1 in retina. In normal adult retina, PARP-1 was mildly detected in the cytoplasm of the GCL and nerve fiber layer (Fig. 5A). There were markedly fewer PARP-1 positive cells in the IR injury groups at 7 days. However, the immunoreactivity of PARP-1 was concentrated predominantly in the nuclei of the GCL in IR retina (Fig. 5C). This altered location of PARP-1 from the cytoplasm to the nuclei was previously observed by Huang et al (24) in the same rodent experiment model. Since the cleaved $29 \mathrm{kDa}$ fragment of PARP-1 can translocate to the nuclei and bind to the oxidized DNA breaks, the translocation of PARP-1 immunoreactivity illustrated the sustained existence of DNA breaks in RIR injury. By contrast, after 7 days of intraperitoneal administration of HRS in IR rats, there was apparent immunoreactivity of PARP-1 not only in the nuclei but also in the cytoplasm of the GCL as well as in the nerve fiber layer (Fig. 5D).

\section{Discussion}

DNA damage due to oxidative stress is a crucial factor in the pathogenesis of neurodegenerative diseases (25). It has been demonstrated that DNA oxidation is involved in the pathophysiology of retinal disorder $(4,26)$, including RIR injury, which typically manifests as ganglion cell degeneration. Peroxynitrite, generated from $\mathrm{NO}$ and $\mathrm{O}_{2-}$, destroys lipid, protein and nucleic acid and is considered to be the most effective promoter of cell death $(27,28)$. Since peroxynitrite induces the selective oxidation of guanine in DNA, its oxidative product, $8-\mathrm{OHdG}$, is the most widely used 'marker' for oxidative DNA damage (29-32). 
Activation of PARP-1 is recognized to perform central roles in DNA repair, as its DNA binding domain may seal the breaks on DNA strands and prevent incorrect DNA recombination (33). However, severely sustained DNA injury induces overactivation of PARP-1, which in turn leads to increased PAR production that augments the translocation of AIF into the nuclei to induce DNA cleavage into large fragments. This process is known as PARP-1-mediated cell death (PARthanatos) (34-37). Furthermore, the overproduction of PAR can cause lethal consumption of cellular $\mathrm{NAD}^{+}$, consequently leading to energy exhausted cell death (37).

In addition, oxidative stress can also cause mitochondria and endoplasmic reticulum to trigger cell apoptosis. For instance, oxidative stress leads to the opening of mitochondrial membrane permeability transition pores and the release of cytochrome $c$, followed by the activation of caspase- 3 in the form of cleaved fragments. Since activated caspase- 3 has the potential to cleave PARP-1, cell death may be partially inhibited due to its inhibition of PARP-1 activity. Simultaneously, cleaved caspase-3 induces apoptosis, which may be cellular compensation for the severe destruction caused by PARthanatos $(38,39)$. Therefore, PARP-1 and caspase-3 may be simultaneously involved in the procedure and affect each other mutually.

The powerful reductive potential of hydrogen in medicine has been recently recognized $(14,16-18)$. It selectively neutralizes peroxynitrite and hydroxyl radicals generated from oxidative stress and promotes cell survival in vitro and in vivo $(14,40,41)$. Furthermore, HRS is more conveniently used in vivo. The present study demonstrated that peritoneal administration of HRS successfully promoted cell survival in the GCL in a rat RIR model. This result was in accordance with the results of the study by Oharazawa et al in which hydrogen loaded eyedrops reduced TUNEL positive cells in nuclear layers of an RIR model (42).

DNA oxidation was significantly reduced following administration of HRS, demonstrating that molecular hydrogen successfully rescued cells by preventing DNA oxidative breaks. Further investigation of the expression of PARP-1 fragments demonstrated its elevated cleavage from the caspase family following IR injury, which was further confirmed by the condensed nuclear aggregation of intact PARP-1 and its $29 \mathrm{kDa}$ cleaved fragment in the GCL in rodent RIR. This result illustrates the overactivation of PARP-1 can cause cell death in RIR. Notably, following treatment with hydrogen, the integrity of PARP-1 was significantly well preserved, suggesting the recovery of its repairing function to maintain stability of neurons and endothelia in retinas.

In addition, caspase-3 cleavage was significantly elevated following IR injury whereas hydrogen intervention reduced caspase-3 cleavage, suggesting that caspase-mediated apoptosis is inhibited by molecular hydrogen.

In terms of oxidative injury in retina, there are several studies which support that PARP-1-mediated cell death may be an important mechanism of RIR injury. Li et al (43) found that hydrogen peroxide, a strong oxidizer, induced RGC-5 cell death, which induced abundant production of ROS. The authors found that there was no activation of caspase-3 following oxidative injury, however, PARP and its downstream effector, AIF, were involved (43). Furthermore, Li et al (44) investigated
D-galactose-induced oxidative stress in neuroblastoma cells and observed significant necrotic cell death, which could not be alleviated by the caspase inhibitor z-VAD-fmk, suggesting that caspase-dependent cell death did not occur. It is possible that PARP-1-mediated cell death co-exists with caspase-dependent apoptosis in RIR injury and molecular hydrogen can modulate these two procedure simultaneously in neuroretina.

Considering the data presented, hydrogen-mediated neuroprotection in the rat retina may result from inhibition of PARP-1. The histological findings strengthen these results, providing morphological evidence of RGC survival. However, further studies on apoptosis and PARthanatos are required in order to confirm the mechanism of hydrogen intervention.

\section{Acknowledgements}

The authors would like to thank Professor Yongming Wang and Professor Jinyan He (Tianjin Medical University) for their assistance with the animal procedures and for consultation on the experimental technique.

This study was supported by research grants from the National Natural Science Foundation of China (nos. 81071533 and 81101409), Tianjin Municipal Science and Technology Commission Foundation (nos. 11JCYBJC12900 and 13JCQNJC11400), the Foundation of Tianjin Bureau of Public Health (no. 2011KZ108) and the Foundation of Tianjin Municipal Education Commission (no. 20120129).

\section{References}

1. Bringmann A, Uckermann O, Pannicke T, Iandiev I, Reichenbach A and Wiedemann P: Neuronal versus glial cell swelling in the ischaemic retina. Acta Ophthalmol Scand 83: 528-538, 2005.

2. Sun MH, Pang JH, Chen SL, Han WH, Ho TC, Chen KJ, Kao LY, Lin KK and Tsao YP: Retinal protection from acute glaucoma-induced ischemia-reperfusion injury through pharmacologic induction of heme oxygenase-1. Invest Ophthalmol Vis Sci 51: 4798-4808, 2010.

3. Makita J, Hosoya K, Zhang P and Kador PF: Response of rat retinal capillary pericytes and endothelial cells to glucose. J Ocul Pharmacol Ther 27: 7-15, 2011.

4. Liu Y, Tang L and Chen B: Effects of antioxidant gene therapy on retinal neurons and oxidative stress in a model of retinal ischemia/reperfusion. Free Radic Biol Med 52: 909-915, 2012.

5. Barakat DJ, Dvoriantchikova G, Ivanov D and Shestopalov VI: Astroglial $\mathrm{NF}-\kappa \mathrm{B}$ mediates oxidative stress by regulation of NADPH oxidase in a model of retinal ischemia reperfusion injury. J Neurochem 120: 586-597, 2012.

6. Pazdro R and Burgess JR: Differential effects of $\alpha$-tocopherol and $\mathrm{N}$-acetyl-cysteine on advanced glycation end product-induced oxidative damage and neurite degeneration in SH-SY5Y cells. Biochim Biophys Acta 1822: 550-556, 2012.

7. Poulsen HE, Specht E, Broedbaek K, Henriksen T, Ellervik C, Mandrup-Poulsen T, Tonnesen M, Nielsen PE, Andersen HU and Weimann A: RNA modifications by oxidation: A novel disease mechanism? Free Radic Biol Med 52: 1353-1361, 2012.

8. Jaeschke H, McGill MR and Ramachandran A: Oxidant stress, mitochondria and cell death mechanisms in drug-induced liver injury: lessons learned from acetaminophen hepatotoxicity. Drug Metab Rev 44: 88-106, 2012.

9. Virág L and Szabó C: The therapeutic potential of poly (ADP-ribose) polymerase inhibitors. Pharmacol Rev 54: 375-429, 2002.

10. Jagtap P and Szabó C: Poly (ADP-ribose) polymerase and the therapeutic effects of its inhibitors. Nat Rev Drug Discov 4: 421-440, 2005.

11. Hassa PO and Hottiger MO: The diverse biological roles of mammalian PARPS, a small but powerful family of poly-ADP-ribose polymerases. Front Biosci 13: 3046-3082, 2008. 
12. Rodrigues FP, Pestana CR, Polizello AC, Pardo-Andreu GL, Uyemura SA, Santos AC, Alberici LC, da Silva RS and Curti C: Release of NO from a nitrosyl ruthenium complex through oxidation of mitochondrial NADH and effects on mitochondria. Nitric Oxide 26: 174-181, 2012.

13. Dole M, Wilson FR and Fife WP: Hyperbaric hydrogen therapy: a possible treatment for cancer. Science 190: 152-154, 1975.

14. Nagata K, Nakashima-Kamimura N, Mikami T, Ohsawa I and Ohta S: Consumption of molecular hydrogen prevents the stress-induced impairments in hippocampus-dependent learning tasks during chronic physical restraint in mice. Neuropsychopharmacology 34: 501-508, 2009.

15. Terasaki Y, Ohsawa I, Terasaki M, Takahashi M, Kunugi S, Dedong K, Urushiyama H, Amenomori S, Kaneko-Togashi M, Kuwahara N, Ishikawa A, et al: Hydrogen therapy attenuates irradiation-induced lung damage by reducing oxidative stress. Am J Physiol Lung Cell Mol Physiol 301: L415-L426, 2011.

16. Cai J, Kang Z, Liu K, Liu W, Li R, Zhang JH, Luo X and Sun X: Neuroprotective effects of hydrogen saline in neonatal hypoxia-ischemia rat model. Brain Res 1256: 129-137, 2009.

17. Spulber S, Edoff K, Hong L, Morisawa S, Shirahata S and Ceccatelli S: Molecular hydrogen reduces LPS-induced neuroinflammation and promotes recovery from sickness behaviour in mice. PLoS One 7: e42078, 2012.

18. Ohsawa I, Ishikawa M, Takahashi $\mathrm{K}$, Watanabe M, Nishimaki K, Yamagata K, Katsura K, Katayama Y, Asoh S and Ohta S: Hydrogen acts as a therapeutic antioxidant by selectively reducing cytotoxic oxygen radicals. Nat Med 13: 688-694, 2007.

19. Fischer D, Pavlidis M and Thanos S: Cataractogenic lens injury prevents traumatic ganglion cell death and promotes axonal regeneration both in vivo and in culture. Invest Ophthalmol Vis Sci 41: 3943-3954, 2000.

20. Hughes WF: Quantitation of ischemic damage in the rat retina. Exp Eye Res 53: 573-582, 1991.

21. Sellés-Navarro I, Villegas-Pérez MP, Salvador-Silva M, Ruiz-Gómez JM and Vidal-Sanz M: Retinal ganglion cell death after different transient periods of pressure-induced ischemia and survival intervals. A quantitative in vivo study. Invest Ophthalmol Vis Sci 37: 2002-2014, 1996.

22. Biermann J, Lagrèze WA, Dimitriu C, Stoykow C and Goebel U: Preconditioning with inhalative carbon monoxide protects rat retinal ganglion cells from ischemia/reperfusion injury. Invest Ophthalmol Vis Sci 51: 3784-3791, 2010.

23. Hirooka K, Miyamoto O, Jinming P, Du Y, Itano T, Baba T, Tokuda M and Shiraga F: Neuroprotective effects of D-allose against retinal ischemia-reperfusion injury. Invest Ophthalmol Vis Sci 47: 1653-1657, 2006.

24. Huang W, Dobberfuhl A, Filippopoulos T, Ingelsson M, Fileta JB, Poulin NR and Grosskreutz CL: Transcriptional up-regulation and activation of initiating caspases in experimental glaucoma. Am J Pathol 167: 673-681, 2005.

25. Hegde ML, Mantha AK, Hazra TK, Bhakat KK, Mitra S and Szczesny B: Oxidative genome damage and its repair: implications in aging and neurodegenerative diseases. Mech Ageing Dev 133: 157-168, 2012

26. Ozawa Y, Sasaki M, Takahashi N, Kamoshita M, Miyake S and Tsubota K: Neuroprotective effects of lutein in the retina. Curr Pharm Des 18: 51-56, 2012.

27. Guidarelli A, Cerioni L, Tommasini I, Brüne B and Cantoni O: A downstream role for protein kinase Calpha in the cytosolic phospholipase A2-dependent protective signalling mediated by peroxynitrite in U937 cells. Biochem Pharmacol 69: 1275-1286, 2005 .
28. Ali TK, Matragoon S, Pillai BA, Liou GI and El-Remessy AB: Peroxynitrite mediates retinal neurodegeneration by inhibiting nerve growth factor survival signaling in experimental and human diabetes. Diabetes 57: 889-898, 2008

29. Tuo J, Liu L, Poulsen HE, Weimann A, Svendsen O and Loft S: Importance of guanine nitration and hydroxylation in DNA in vitro and in vivo. Free Radic Biol Med 29: 147-155, 2000

30. Whiteman M, Hong HS, Jenner A and Halliwell B: Loss of oxidized and chlorinated bases in DNA treated with reactive oxygen species: implications for assessment of oxidative damage in vivo. Biochem Biophys Res Commun 296: 883-889, 2002.

31. Haider L, Fischer MT, Frischer JM, Bauer J, Höftberger R, Botond G, Esterbauer H, Binder CJ, Witztum JL and Lassmann $\mathrm{H}$ : Oxidative damage in multiple sclerosis lesions. Brain 134: 1914-1924, 2011.

32. Werner SR, Prahalad AK, Yang J and Hock JM: RECQL4-deficient cells are hypersensitive to oxidative stress/damage: Insights for osteosarcoma prevalence and heterogeneity in Rothmund-Thomson syndrome. Biochem Biophys Res Commun 345: 403-409, 2006.

33. Chaitanya GV, Steven AJ and Babu PP: PARP-1 cleavage fragments: signatures of cell-death proteases in neurodegeneration. Cell Commun Signal 8: 31, 2010.

34. Dantzer F, de La Rubia G, Ménissier-de Murcia J, Hostomsky Z, de Murcia G and Schreiber V: Base excision repair is impaired in mammalian cells lacking Poly (ADP-ribose) polymerase-1. Biochemistry 39: 7559-7569, 2000.

35. Harraz MM, Dawson TM and Dawson VL: Advances in neuronal cell death 2007. Stroke 39: 286-288, 2008

36. Wang Y, Dawson VL and Dawson TM: Poly (ADP-ribose) signals to mitochondrial AIF: a key event in parthanatos. Exp Neurol 218: 193-202, 2009

37. Wang Y, Kim NS, Haince JF, Kang HC, David KK, Andrabi SA, Poirier GG, Dawson VL and Dawson TM: Poly (ADP-ribose) (PAR) binding to apoptosis-inducing factor is critical for PAR polymerase-1-dependent cell death (parthanatos). Sci Signal 4: ra20, 2011

38. Kliem H, Berisha B, Meyer HH and Schams D: Regulatory changes of apoptotic factors in the bovine corpus luteum after induced luteolysis. Mol Reprod Dev 76: 220-230, 2009.

39. Mei YP, Zhou JM, Wang Y, Huang H, Deng R, Feng GK, Zeng YX and Zhu XF: Silencing of LMP1 induces cell cycle arrest and enhances chemosensitivity through inhibition of AKT signaling pathway in EBV-positive nasopharyngeal carcinoma cells. Cell Cycle 6: 1379-1385, 2007.

40. Cardinal JS, Zhan J, Wang Y, Sugimoto R, Tsung A, McCurry KR, Billiar TR and Nakao A: Oral hydrogen water prevents chronic allograft nephropathy in rats. Kidney Int 77: 101-109, 2010.

41. Hanaoka T, Kamimura N, Yokota T, Takai S and Ohta S: Molecular hydrogen protects chondrocytes from oxidative stress and indirectly alters gene expressions through reducing peroxynitrite derived from nitric oxide. Med Gas Res 1: 18, 2011.

42. Oharazawa H, Igarashi T, Yokota T, Fujii H, Suzuki H, Machide M, Takahashi $\mathrm{H}$, Ohta S and Ohsawa I: Protection of the retina by rapid diffusion of hydrogen: administration of hydrogen-loaded eye drops in retinal ischemia-reperfusion injury. Invest Ophthalmol Vis Sci 51: 487-492, 2010.

43. Li GY and Osborne NN: Oxidative-induced apoptosis to an immortalized ganglion cell line is caspase independent but involves the activation of poly (ADP-ribose) polymerase and apoptosis-inducing factor. Brain Res 1188: 35-43, 2008

44. Li N, He Y, Wang L, Mo C, Zhang J, Zhang W, Li J, Liao Z, Tang $X$ and Xiao H: D-galactose induces necroptotic cell death in neuroblastoma cell lines. J Cell Biochem 112: 3834-3844, 2011. 